\title{
C-Mannosyl tryptophan increases in the plasma of patients with ovarian cancer
}

\author{
NAOYUKI IWAHASHI ${ }^{1}$, YOKO INAI $^{2}$, SHIHO MINAKATA $^{2}$, SHO SAKURAI $^{2}$, \\ SHINO MANABE ${ }^{3}$, YUKISHIGE ITO ${ }^{3}$, KAZUHIKO INO $^{1}$ and YOSHITO IHARA ${ }^{2}$
}

\begin{abstract}
Departments of ${ }^{1}$ Obstetrics and Gynecology, and ${ }^{2}$ Biochemistry, Wakayama Medical University, Wakayama 641-0012; ${ }^{3}$ Synthetic Cellular Chemistry Laboratory, RIKEN (The Institute of Physical and Chemical Research), Saitama 351-0198, Japan
\end{abstract}

Received June 18, 2019; Accepted October 16, 2019

DOI: $10.3892 / \mathrm{ol} .2019 .11161$

\begin{abstract}
Ovarian cancer survival is poor, in part, because there are no specific biomarkers for early diagnosis. C-Mannosyl tryptophan (CMW) is a structurally unique glycosylated amino acid recently identified as a novel biomarker of renal dysfunction. The present study investigated whether blood CMW is altered in patients with ovarian cancer and whether differences in blood CMW can distinguish benign from malignant ovarian tumors. Plasma samples were obtained from 49 patients with malignant, borderline or benign ovarian tumors as well as from seven age-matched healthy women. CMW was identified and quantified in these samples using ultra-performance liquid chromatography with fluorometry. Plasma CMW was significantly higher in the malignant tumor group than in the borderline and benign tumor groups, and higher in the combined tumor group (malignant, borderline or benign) compared with healthy controls. Receiver operating characteristic curve analysis of plasma CMW distinguished malignant tumors from borderline/benign tumors [area under the curve $(\mathrm{AUC})=0.905]$. Discrimination performance was greater than that of cancer antigen $(\mathrm{CA}) 125$ (AUC=0.835), and CMW + CA125 combined achieved even greater discrimination $(\mathrm{AUC}=0.913,81.8 \%$ sensitivity, $87.5 \%$ specificity, $93.1 \%$ positive predictive value and $70.0 \%$ negative predictive value) Plasma CMW differentiates malignant ovarian cancer from borderline or benign ovarian tumors with high accuracy, and performance is further improved by combined CMW and CA125 measurement.
\end{abstract}

Correspondence to: Dr Naoyuki Iwahashi, Department of Obstetrics and Gynecology, Wakayama Medical University, 811-1 Kimiidera, Wakayama 641-0012, Japan

E-mail:naoyuki@wakayama-med.ac.jp

Professor Yoshito Ihara, Department of Biochemistry, Wakayama Medical University, 811-1 Kimiidera, Wakayama 641-0012, Japan E-mail: y-ihara@wakayama-med.ac.jp

Key words: epithelial ovarian cancer, glycosylation, C-mannosyl tryptophan, cancer antigen 125 , tumor marker

\section{Introduction}

Ovarian cancer remains the most lethal gynecologic disease as it is often diagnosed at an advanced stage, and little progress has been achieved in chemotherapy treatment $(1,2)$. Early differential diagnosis and timely treatment are essential because mortality is closely related to disease stage, with 5-year survival dramatically higher when detected in stage I or II (70\%) than when detected in stage III (40\%) and stage IV (20\%) (3). Imaging modalities such as transvaginal ultrasonography (US), computed tomography (CT), and magnetic resonance imaging are critical for diagnosis and preoperative management because biopsy is not feasible. However, these imaging modalities may be insufficient to provide a correct preoperative diagnosis of malignancy (4). Alternatively, 18F-fluoro-2-deoxy-D-glucose positron emission tomography combined with CT (FDG-PET/CT) is a highly effective imaging modality for diagnosis and treatment monitoring of ovarian cancer (5), but is too expensive for routine screening of all patients with pelvic masses. For blood screening of ovarian cancer, cancer antigen 125 (CA125) is the primary diagnostic biomarker for detection of malignancy. However, blood CA125 increases not only in borderline and malignant ovarian tumor patients but also in benign cases and even under certain physiological and pathological conditions such as menses, pregnancy, endometriosis, and peritoneum inflammatory diseases $(4,6)$. Efforts have been made to improve early screening and differential diagnosis by combining serum CA125 measurement with transvaginal US, but sensitivity and specificity are still insufficient for reliable prediction of malignancy $(4,7,8)$. Thus, to further improve ovarian cancer survival, additional cancer-specific diagnostic biomarkers are required for more reliable early detection and diagnosis.

C-Mannosyl tryptophan (CMW) is a glycosylated amino acid first isolated from human urine (9) with a unique glycan structure in which an $\alpha$-mannose is bound to the indole $\mathrm{C} 2$ carbon of a Trp residue through a C-C linkage (10). CMW was also identified in human ribonuclease 2 (RNase2) as a post-translational modification (11). C-Mannosylation at the first $\operatorname{Trp}$ in the consensus amino acid sequence $\operatorname{Trp}-\mathrm{X}-\mathrm{X}-\mathrm{Tr} \mathrm{p} / \mathrm{Cys}$ of proteins is catalyzed by a specific C-mannosyltransferase $(12,13)$. The genes encoding mammalian C-mannosyltransferase (DPY19L1, L3) were 
identified based on homology to the Caenorhabditis elegans DPY19 gene (13-15). The consensus sequence is frequently C-mannosylated in proteins of the thrombospondin type 1 repeat (TSR) superfamily and type I cytokine receptor family (16). However, the pathway for generation of the CMW monomer is still unknown.

In regard to human health and protein C-mannosylation, it was reported that blood CMW is elevated in patients with renal dysfunction, including renal diseases associated with type 2 diabetes (17-21). As for cancer biology, it was recently reported that $\mathrm{C}$-mannosylation of $\mathrm{R}$-spondin 2 activates $\mathrm{Wnt} / \beta$-catenin signaling and migration activity in various human tumor cells (22). This study suggested that $\mathrm{C}$-mannosylation of $\mathrm{R}$-spondin 2 is involved in the promotion of cancer progression. Furthermore, spondin 2 (mindin), a substrate protein for C-mannosylation (23), is increased in the blood of ovarian cancer patients (24). These studies suggest that protein $\mathrm{C}$-mannosylation and $\mathrm{CMW}$ may be involved in the pathophysiological processes of cancer progression. However, there have been no reports on changes in blood CMW in patients with cancer. Recently, we established a novel CMW assay using ultra-performance liquid chromatography (UPLC) and found that the tissue level of CMW is especially high in mouse ovary, uterus, and testis (25). Thus, in the present study, we applied our novel assay method to biological samples from ovarian cancer patients to examine the possible utility of CMW for the diagnosis or staging of ovarian cancer.

\section{Materials and methods}

Patient selection and sample collection. Patients treated surgically for benign gynecological disease, benign ovarian tumor, borderline ovarian tumor, or malignant ovarian cancer at Wakayama Medical University Hospital from January 2015 to January 2019 were included in this study. The data of age, clinical stage, histological subtype, serum CA125, serum carbohydrate antigen 19-9 (CA19-9), serum creatinine, and maximum cyst diameter were extracted from patients' medical record files and analyzed. To remove the effects of renal function on CMW, patients with renal dysfunction (serum creatinine $\geq 1.0 \mathrm{mg} / \mathrm{dl}$ ) were excluded from the study. Histological diagnosis was determined on the basis of standard hematoxylin and eosin (H\&E)-stained sections by two or more experienced senior pathologists according to the criteria of the World Health Organization (WHO). Tumor staging was conducted according to the International Federation of Gynecology and Obstetrics (FIGO) classification. Blood samples were obtained from all patients as well as from seven age-matched healthy controls. In addition to pre-treatment plasma samples, post-treatment plasma samples were obtained from three of the advanced malignant cancer patients at the point of interval debulking surgery 28 days after neoadjuvant chemotherapy (NAC) including three cycles of paclitaxel $\left(175 \mathrm{mg} / \mathrm{m}^{2}\right)$ and carboplatin (AUC: 5.0 , Calvert's formula). The study was approved by the ethics committee of Wakayama Medical University (authorization number: 1825) and was conducted in accordance with the tenets of the Declaration of Helsinki. All patients in this study provided written informed consent for the use of their plasma and tissue samples.
Materials. The reagents used in the study were obtained from Sigma-Aldrich, Japan, Waters Corporation, or FUJIFILM Wako Pure Chemical Corporation.

Sample preparation for CMW analysis. Blood samples were collected in ethylenediaminetetraacetic acid (EDTA) tubes and centrifuged at 2,000 x g for $10 \mathrm{~min}$ to obtain plasma. Ovarian tissue specimens were collected immediately after surgical excision. The samples were frozen and stored at $-80^{\circ} \mathrm{C}$ until use. For measurement of CMW, the plasma samples were diluted in extraction solution (methanol:acetonitrile:formic acid=50:49.9:0.1) and centrifuged at 12,000 x g for $15 \mathrm{~min}$ at $4{ }^{\circ} \mathrm{C}$. The ovarian tissue samples were homogenized in the same extraction solution, and the supernatants were similarly collected. All supernatants were further filtered using a $0.45-\mu \mathrm{m}$ polyvinylidene difluoride (PVDF) syringe filter before liquid chromatography analysis.

Synthesis of C2- $\alpha$-C-mannosyl-L-tryptophan. C2- $\alpha-\mathrm{C}-$ mannosyl-L-tryptophan (CMW) was synthesized as previously described (26). The purity and identity of the final product were verified by ${ }^{1} \mathrm{H}$ NMR spectroscopy and matrix-assisted laser desorption ionization (MALDI) mass spectrometry. The proton chemical shifts and coupling constants were consistent with those reported previously, and the mass on MALDI mass spectrometry was consistent with the expected mass of the correct product.

Ultra-performance liquid chromatography conditions to analyze CMW. CMW in biological samples was analyzed and quantified by chromatographic assay as previously described (25). The samples were injected into a Waters Acquity UPLC H-Class system (Waters Corporation) equipped with an Acquity UPLC BEH Amide column, photodiode array detector, fluorescence detector. CMW was quantified as described (25) by measuring the fluorescence (excitation at $285 \mathrm{~nm} / \mathrm{emission}$ at $350 \mathrm{~nm})$ or mass abundance $(\mathrm{m} / \mathrm{z}$ value of $367.15[\mathrm{M}+\mathrm{H}]^{+}$for $\left.\mathrm{CMW}\right)$. Empower 3 software was used to collect and process data.

Chemically synthesized CMW was used as a standard compound in the assays. The detection limit of CMW based on the measured fluorescence was $2 \mathrm{nM}$. Extraction recovery rates were evaluated by comparing plasma and ovary tissue samples prepared as described to samples including a known amount of CMW. The extraction recovery rate of plasma CMW detected by fluorescence was $90.9 \%$.

Statistical analysis. Statistical analyses were performed using JMP Pro version 13.1.0 for Windows (SAS Institute Inc.). Statistical comparisons between the groups were performed using the Spearman's rank correlation coefficient, the Kruskal-Wallis test, the Steel-Dwass test, or Mann-Whitney U test as appropriate. $\mathrm{P}<0.05$ (two-tailed) was considered significant for all tests. Receiver operating characteristic (ROC) curves were constructed, and area under the curve (AUC) were calculated to evaluate the utility of CMW, CA125, and combined CMW + CA125 to discriminate among borderline ovarian tumor, malignant ovarian cancer, and benign ovarian tumor. We developed a CMW + CA125 combined prediction model based on logistic regression with the best subset 
selection method. The ROC curves were also used to determine the best cut-off value for CMW, CA125, and CMW + CA125 combined, which was defined as the point situated farthest from the reference line. According to these cut-off values, sensitivity, specificity, positive predictive value (PPV), and negative predictive value (NPV) were calculated.

\section{Results}

Clinical characteristics of study cohort. The characteristics of the patients are shown in Table 1. Plasma samples were obtained from seven age-matched healthy women (used as normal controls), eight patients with benign ovarian tumor, eight with borderline ovarian tumor, and 33 patients with malignant ovarian cancer. There were no significant differences in age, serum creatinine, and maximum cyst diameter among the plasma sample groups.

Surgically resected ovarian tissue samples were also obtained from nine patients with other benign gynecological diseases (used as normal controls), 16 patients with benign ovarian tumors, 16 with borderline ovarian tumors, and 20 patients with malignant ovarian cancer (Table 1).

Detection and quantification of CMW in human sample.Plasma and tissue CMW was detected and quantified using UPLC with fluorescence intensity as described in Materials and Methods. The human plasma samples from controls and patients were separated by Hydrophilic Interaction Liquid Chromatography (HILIC), and CMW (arrow in Fig. 1A) was detected by monitoring the fluorescence (excitation at $285 \mathrm{~nm} / \mathrm{emission}$ at $350 \mathrm{~nm}$ ). The typical elution pattern of CMW is shown in Fig. 1A. CMW was detected as the main fluorescence peak at $5.2 \mathrm{~min}$. The target peak was further confirmed as CMW by adding chemically synthesized CMW (26) (Sample + CMW in Fig. 1A) to a subset of samples. The mass of the target peak was confirmed as CMW by mass spectrometry $(\mathrm{m} / \mathrm{z}$ value of $\left.367.15[\mathrm{M}+\mathrm{H}]^{+}\right)$in a part of the samples. The mass abundance was also measured (data not shown). The amount of CMW was quantified in the biological samples using a calibration curve constructed from chemically synthesized CMW as described previously (25). In ovarian tissue samples (20 malignant, 16 borderline, 16 benign, and 9 normal), there were no significant differences in tissue CMW among each of the groups ( $\mathrm{P}=0.972$; Fig. 1B).

Comparison of plasma CMW and serum CA125 among malignant, borderline, and benign tumors. The plasma CMW and serum CA125 levels for all 49 patients with malignant, borderline, or benign tumors as well as seven healthy normal controls are shown in Fig. 2. Plasma CMW was significantly higher in the malignant (median: $333.6 \mathrm{nM}$ ), borderline (median: $208.5 \mathrm{nM}$ ), and benign (median: $174.9 \mathrm{nM}$ ) tumor groups compared to the normal control group (median: $148.1 \mathrm{nM}$; Fig. 2A) and significantly higher in the malignant tumor group than the borderline and benign tumor groups. Serum CA125 also differed significantly among the three tumor groups (Fig. 2B) but was not correlated with plasma CMW (Fig. 2C). In the malignant tumor group, there were no significant differences in plasma CMW (Fig. 2D) or serum CA125 (Fig. 2E) among FIGO stages. There was also no correlation between plasma CMW and serum CA125 in the malignant tumor group (Fig. 2F).

Diagnostic performance of plasma CMW among malignant, borderline, and benign tumors. ROC curves of plasma CMW and serum CA125 were generated to assess the utility of these markers to discriminate among patients with malignant ovarian cancer, borderline ovarian tumor, and benign ovarian tumor (Fig. 3). To distinguish malignant/borderline tumors from benign tumors, different cut-off levels of CMW, CA125, and CMW + CA125 combined [prediction model; diagnostic index $=(0.047) \times \mathrm{CMW}+(0.013) \times \mathrm{CA} 125-9.656]$ were evaluated (Fig. 3A-C). Both CMW and CA125 demonstrated reasonable accuracy for distinguishing malignant/borderline from benign tumors (CMW: AUC $=0.927,82.9 \%$ sensitivity, $100.0 \%$ specificity, $100.0 \%$ PPV, and $53.3 \%$ NPV; CA125: AUC $=0.924,97.6 \%$ sensitivity, $75.0 \%$ specificity, $95.2 \%$ PPV, and $85.7 \% \mathrm{NPV})$. However, the combination of both markers yielded a higher AUC $(0.957,82.9 \%$ sensitivity, $100.0 \%$ specificity, $100.0 \% \mathrm{PPV}$, and $53.3 \% \mathrm{NPV}$; $\mathrm{P}<0.05$; Fig. 3A-C). A similar analysis was conducted to examined the efficacy of these markers for distinguishing malignant tumors from borderline/benign tumors with different cut-off levels of CMW, CA125, and CMW + CA125 combined [prediction model; diagnostic index $=(0.022) \times \mathrm{CMW}+(0.001) \mathrm{x}$ CA125-5.376] (Fig. 3D-F). Although the AUC values of both CMW and CA125 were lower (CMW: $A U C=0.905,93.9 \%$ sensitivity, $81.3 \%$ specificity, $91.2 \% \mathrm{PPV}$, and $86.7 \% \mathrm{NPV}$; CA125: AUC $=0.835,69.7 \%$ sensitivity, $93.3 \%$ specificity, 95.8\% PPV, and $60.0 \% \mathrm{NPV}$ ), the AUC of CMW + CA125 was still 0.913 (81.8\% sensitivity, $87.5 \%$ specificity, $93.1 \%$ PPV, and 70.0\% NPV; P<0.05; Fig. 3D-F). Finally, the same methodology was used to assess if these markers alone and combined can distinguish malignant tumors from borderline tumors [prediction model; diagnostic index $=(0.015) \times \mathrm{CMW}$ $+(0.001) \times$ CA125-3.036] (Fig. 3G-I). Although the AUCs of CMW and CA125 were even lower (CMW: $\mathrm{AUC}=0.826$, 93.9\% sensitivity, $62.5 \%$ specificity, $91.2 \%$ PPV, and $71.4 \%$ NPV; CA125: AUC $=0.735,69.7 \%$ sensitivity, $87.5 \%$ specificity, 95.8\% PPV, and $41.2 \% \mathrm{NPV}$ ), the combination still demonstrated superior discrimination performance (AUC $=0.845$, $81.8 \%$ sensitivity, $75.0 \%$ specificity, $93.1 \% \mathrm{PPV}$, and $50.0 \%$ NPV; P<0.05; Fig. 3G-I).

Plasma CMW monitoring for assessing the treatment response. In three ovarian cancer patients, plasma $\mathrm{CMW}$ and $\mathrm{CA} 125$ were measured from both pre-NAC and post-NAC plasma samples to assess the potential for assessment of treatment response (Fig. 4). In NAC-sensitive case 1 (Fig. 4A; serous carcinoma stage III), the sum of the longest diameters (SLD) of the tumor decreased from 21 to $9 \mathrm{~cm}$, and the CA125 level decreased from 8275 to $52.9 \mathrm{U} / \mathrm{ml}$. After surgery, the CA125 level further decreased to $47.2 \mathrm{U} / \mathrm{ml}$. In the plasma samples obtained at the same time points, CMW decreased from $236.9 \mathrm{nM}$ pre-NAC to $223.3 \mathrm{nM}$ post-NAC and then further to $170.8 \mathrm{nM}$ post-surgery. In NAC-sensitive case 2 (Fig. 4B; serous carcinoma stage IV), the SLD of the tumor decreased from 14 to $11 \mathrm{~cm}$, and the CA125 level decreased from 540.2 to $125.7 \mathrm{U} / \mathrm{ml}$. In the plasma samples, CMW decreased from $344.4 \mathrm{nM}$ pre-NAC to $187.9 \mathrm{nM}$ post-NAC. In NAC-resistant case 3 (Fig. 4C; 
Table I. Clinical and pathological characteristics of the study cohort.

A, Malignant ovarian tumor

\begin{tabular}{lcc}
\hline Characteristics & Plasma samples, $\mathrm{n}=33$ & Tissue samples, $\mathrm{n}=20$ \\
\hline Age & $62(30-83)$ & $61(38-79)$ \\
CMW, plasma, nM; tumor, pmol/mg & $333.6(199.4-1615.2)$ & $73.3(30.8-116.2)$ \\
Serum CA125, U/ml & $342.1(13.4-17586.9)$ & - \\
Serum creatinine, mg/dl & $0.59(0.41-0.98)$ & - \\
Maximum cyst diameter, cm & $11(4-21)$ & - \\
Histopathological subtypes, $\mathrm{n}$ & & 10 \\
Serous & 23 & 4 \\
Clear cell & 6 & 4 \\
Mucinous & 2 & 3 \\
Endometrioid & 2 & 8 \\
Stage & & 4 \\
I & 6 & 7 \\
II & 4 & 1 \\
III & 18 & \\
IV & 5 & \\
\hline
\end{tabular}

B, Borderline ovarian tumor

\begin{tabular}{lcr}
\hline Characteristics & Plasma samples, $\mathrm{n}=8$ & Tissue samples, $\mathrm{n}=16$ \\
\hline Age & $57(16-75)$ & $56(35-75)$ \\
CMW, plasma, $\mathrm{nM}$; tumor, pmol/mg & $208.5(161.0-356.2)$ & $71.6(45.6-112.8)$ \\
Serum CA125, U/ml & $95.7(37.5-1343.4)$ & - \\
Serum creatinine, $\mathrm{mg} / \mathrm{dl}$ & $0.58(0.47-0.70)$ & - \\
Maximum cyst diameter, cm & $14.5(3.6-30)$ & - \\
Histopathological subtypes, $\mathrm{n}$ & & 6 \\
Serous & 2 & 7 \\
Mucinous & 3 & 3 \\
Seromucinous & 3 & \\
\hline
\end{tabular}

C, Benign ovarian tumor

\begin{tabular}{lcc}
\hline Characteristics & Plasma samples, $\mathrm{n}=8$ & Tissue samples, $\mathrm{n}=16$ \\
\hline Age & $64(24-77)$ & $58(44-77)$ \\
$\mathrm{CMW}$, plasma, $\mathrm{nM}$; tumor, pmol/mg & $174.9(135.4-220.7)$ & $66.2(42.9-97.0)$ \\
Serum CA12, U/ml & $14.1(5.6-159.5)$ & - \\
Serum creatinin, mg/dl & $0.58(0.42-0.67)$ & - \\
Maximum cyst diameter, cm & $7.9(2.4-14.5)$ & - \\
Histopathological subtypes, $\mathrm{n}$ & & 6 \\
Serous & 3 & 5 \\
Mucinous & 3 & 5 \\
Endometrioid & 2 &
\end{tabular}

D, Normal control

Characteristics

Age

CMW, plasma, nM; tumor, pmol/mg
Plasma samples, $\mathrm{n}=7$

$50(38-54)$

$148.1(134.7-175.2)$
Tissue samples, $\mathrm{n}=9$

48 (46-57)

$71.1(46.7-94.9)$

CMW, C-mannosyl tryptophan. 
A

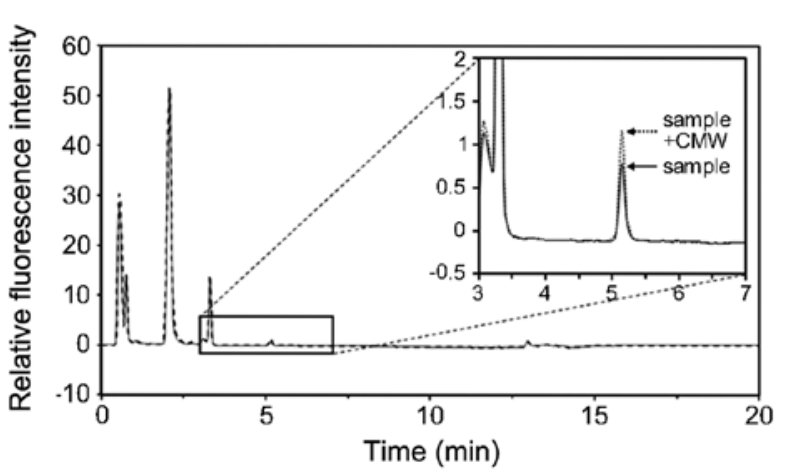

B

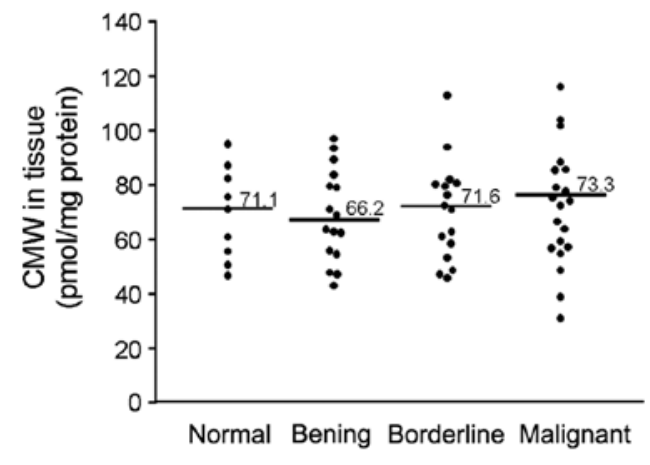

Figure 1. Detection and quantification of CMW in tissue and blood from patients with ovarian cancer. (A) Samples were prepared by organic solvent extraction, followed by centrifugation, and analyzed by UPLC. CMW was quantified by measuring the fluorescence intensity. (B) Tissue CMW levels in the study cohort. Tissue CMW levels were measured and quantified by UPLC, and were indicated as the amounts of CMW (pmol) per total proteins (mg) in the lysate samples. The levels were comparable among patients with malignant, borderline or benign ovarian tumors, and normal controls $(\mathrm{P}=0.972)$. $\mathrm{CMW}, \mathrm{C}-\mathrm{mannosyl}$ tryptophan; UPLC, ultra-performance liquid chromatography.

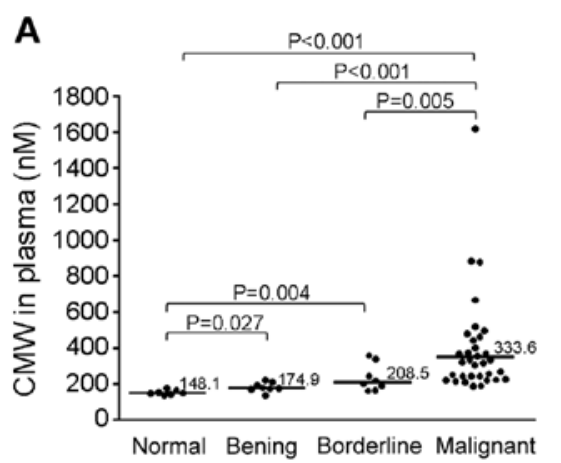

D

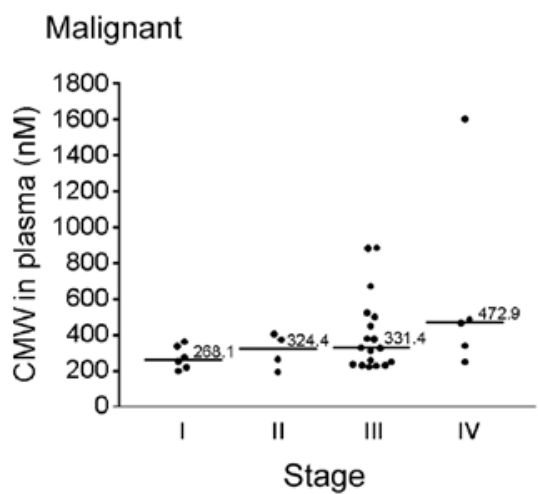

B

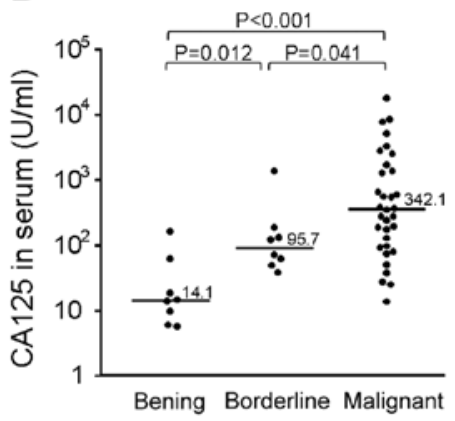

E

Malignant

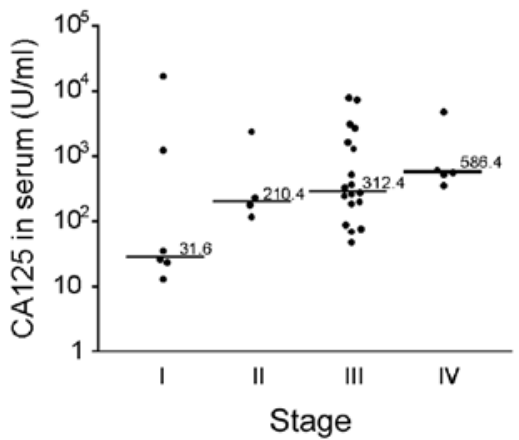

C

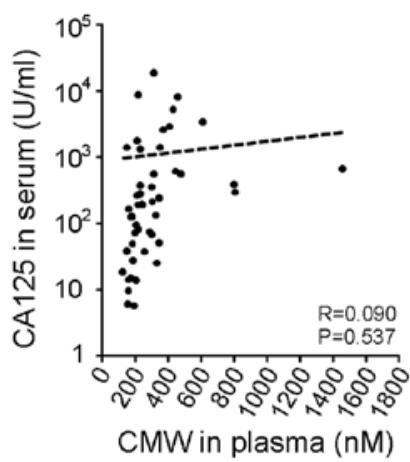

F

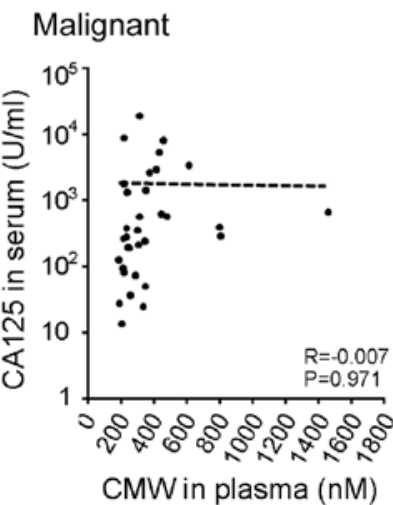

Figure 2. Distribution of plasma CMW and serum CA125 levels in the study cohort. (A) Plasma CMW levels among patients with malignant, borderline or benign ovarian tumors, and normal controls. Plasma $C M W$ was significantly higher in the malignant $(\mathrm{P}<0.001)$, borderline $(\mathrm{P}=0.004)$ and benign $(\mathrm{P}=0.027)$ tumor groups than in the normal control group, and significantly higher in the malignant tumor group than in the borderline $(\mathrm{P}=0.005)$ and benign $(\mathrm{P}<0.001)$ tumor groups. (B) Serum CA125 levels among patients with malignant, borderline or benign ovarian tumors. Serum CA125 was significantly higher in the malignant $(\mathrm{P}<0.001)$ and borderline $(\mathrm{P}=0.012)$ tumor groups than in the benign tumor group, and significantly higher in the malignant tumor group than in the borderline $(\mathrm{P}=0.041)$ tumor group. $(\mathrm{C})$ Correlation analysis between plasma CMW and serum CA125 levels. There were no significant correlations between plasma CMW and serum CA125 levels $(\mathrm{P}=0.537)$. (D) Plasma CMW levels among the malignant group according to FIGO stage. There were no significant differences in plasma CMW among FIGO stages $(\mathrm{P}=0.231)$. (E) Serum CA125 levels among the malignant group according to FIGO stage. There were no significant differences in serum CA125 among FIGO stages $(\mathrm{P}=0.238)$. ( $\mathrm{F})$ Correlation between plasma $\mathrm{CMW}$ and serum $\mathrm{CA} 125$ levels in the malignant group. There were no significant correlations between plasma CMW and serum CA125 levels in the malignant group (P=0.971). CMW, C-mannosyl tryptophan; CA125, cancer antigen 125; FIGO, International Federation of Gynecology and Obstetrics.

mucinous carcinoma stage III), however, the SLD of the tumor increased from 17 to $19 \mathrm{~cm}$, the CA19-9 level increased from
6342 to $8690 \mathrm{U} / \mathrm{ml}$, and plasma CMW increased from $254.8 \mathrm{nM}$ pre-NAC to $314.8 \mathrm{nM}$ post-NAC. 
A

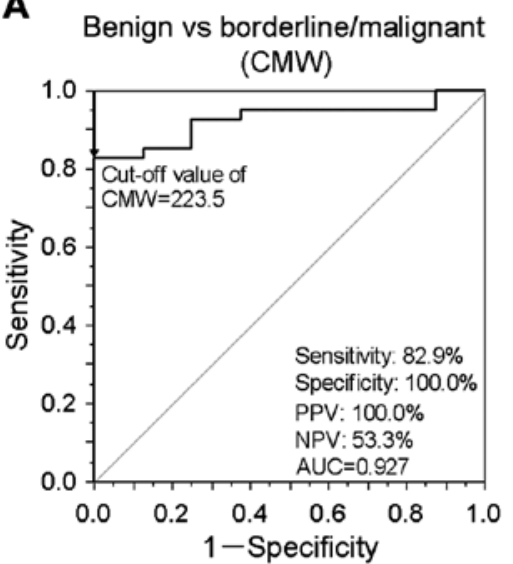

D

Benign/borderline vs malignant (CMW)

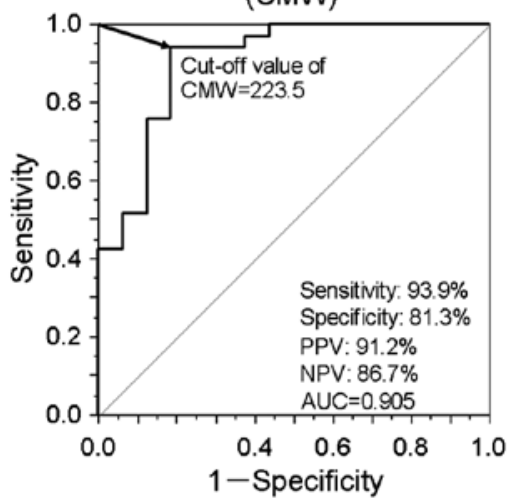

G

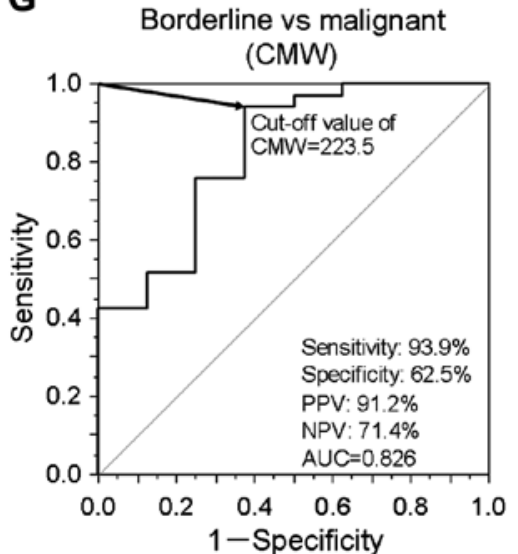

B

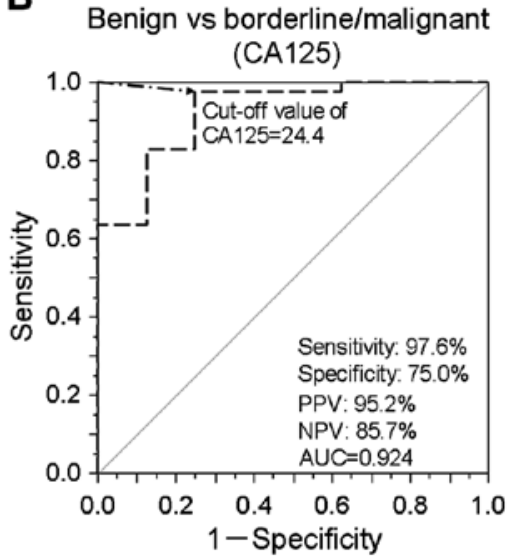

$\mathbf{E}$

Benign/borderline vs malignant (CA125)

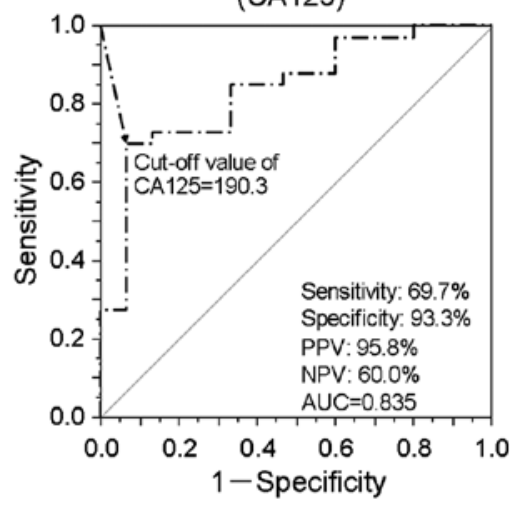

H

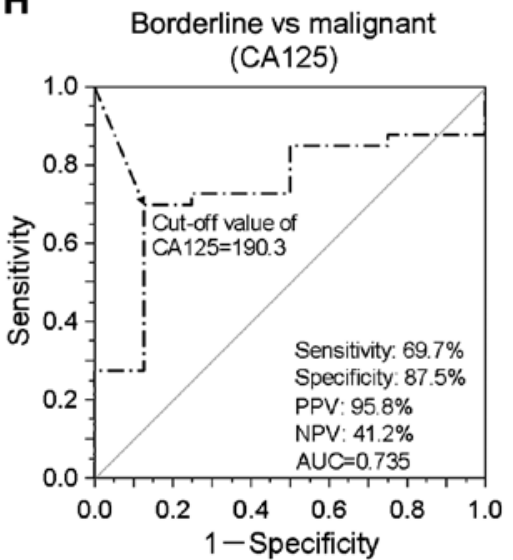

C

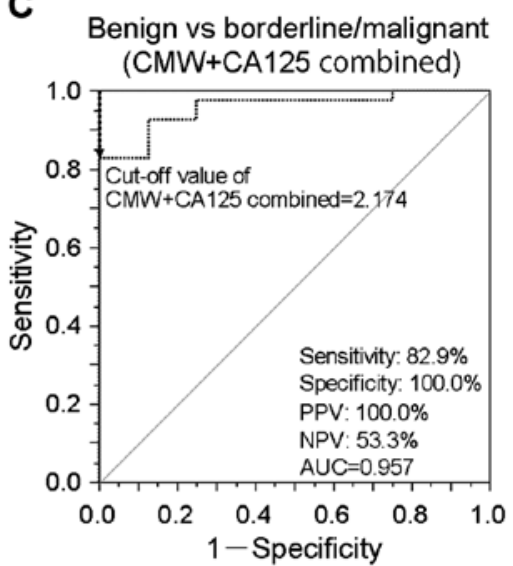

$\mathbf{F}$

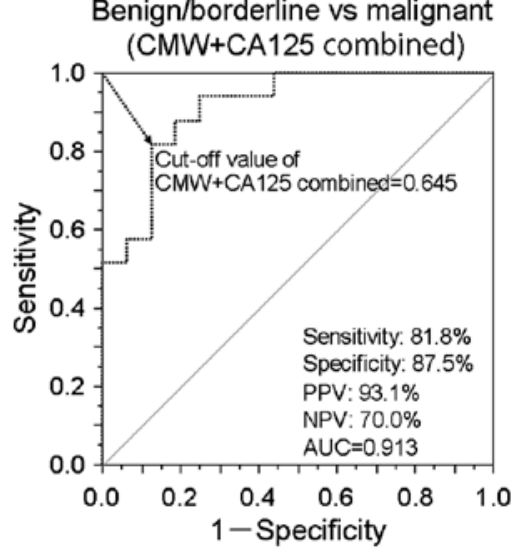

I

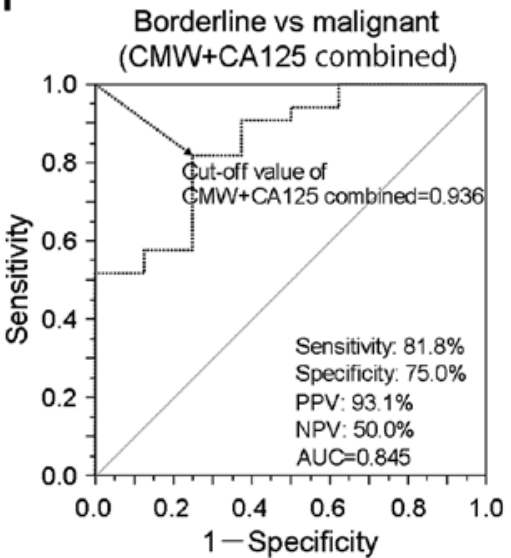

Figure 3. Receiver operating characteristic analyses of plasma CMW and serum CA125 level for distinguishing study groups. Patients with benign vs. borderline/malignant ovarian tumors distinguished according to (A) CMW, (B) CA125 and (C) CMW + CA125 combined. Patients with benign/borderline vs malignant ovarian tumors distinguished according to (D) CMW, (E) CA125 and (F) CMW + CA125 combined. Patients with borderline vs. malignant ovarian tumors distinguished according to (G) CMW, (H) CA125 and (I) CMW + CA125 combined. CMW, C-mannosyl tryptophan; CA125, cancer antigen 125; PPV, positive predictive value; NPV, negative predicative value; AUC, area under the curve.

\section{Discussion}

To the best of our knowledge, this is the first study demonstrating the feasibility of measuring plasma CMW using UPLC to differentiate malignant ovarian cancer from borderline or benign tumors. Indeed, the plasma CMW identified pathologically confirmed malignant ovarian cancer with high sensitivity and specificity, and the combination of plasma CMW with serum CA125 demonstrated even better diagnostic performance than either marker alone. Plasma CMW, alone or in combination with CA125, may allow for early non-invasive screening of ovarian cancer.

Ovarian cancer is often diagnosed at an advanced stage when standard chemotherapies are generally much less effective (1). Although there are several new therapeutic strategies that can be used to treat some cases of ovarian cancer, including targeted therapy involving the vascular endothelial growth factor inhibitor bevacizumab and the poly-(ADP-ribose) 

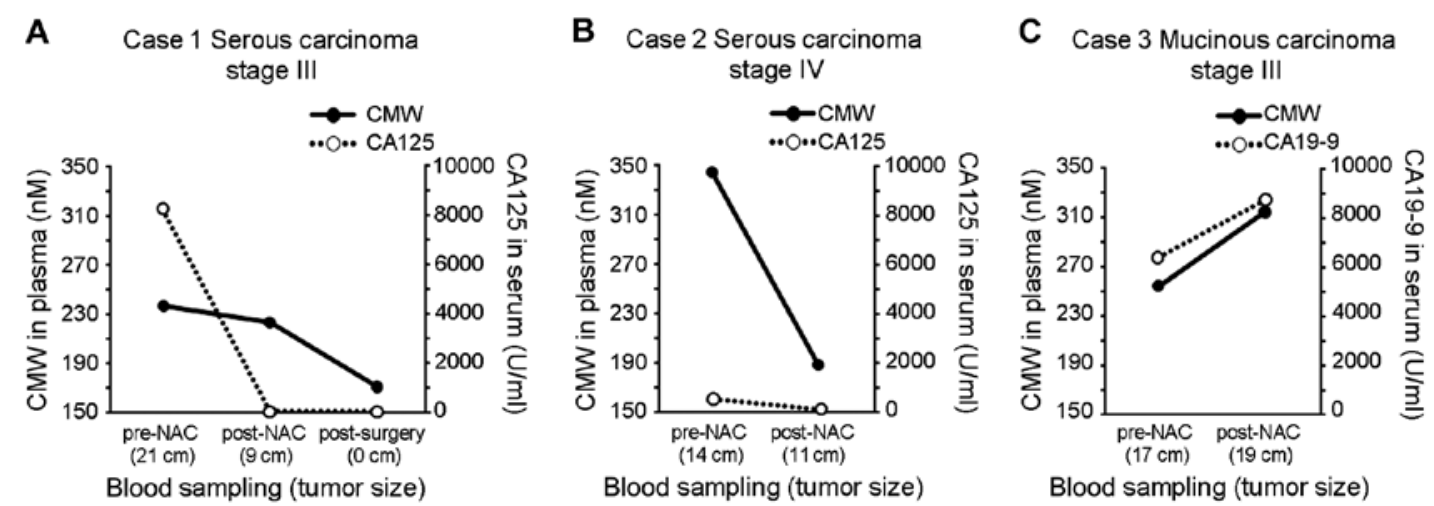

Figure 4. Comparison of plasma CMW and serum CA125 level before and after NAC in two NAC-sensitive cases, (A) case 1 and (B) case 2 , and (C) an NAC-resistant case (case 3). CMW, C-mannosyl tryptophan; CA125, cancer antigen 125; NAC, neoadjuvant chemotherapy.

polymerase inhibitor olaparib $(27,28)$, ovarian cancer is still responsible for the majority of gynecological cancer-related deaths (2). Thus, new treatment strategies, including novel approaches for early diagnosis, tumor monitoring, and tumor targeting, are needed to improve prognosis. Blood biomarkers are attractive for disease screening because they can provide useful information rapidly and cost-effectively without invasive procedures. Serum CA125 is currently considered the most reliable marker to detect epithelial ovarian cancers (29) and a predictor of treatment efficacy (30). Although CA125 has been used as a single biomarker for ovarian cancer $(31,32)$, its specificity is limited. Indeed, a recent large-scale randomized controlled trial assessing the current optimal screening method, CA125 measurement and US, found insignificant mortality reduction (8), underscoring the need for alternative strategies that can detect ovarian cancer at an early stage as well as in asymptomatic women.

Aberrant glycosylation is known to be involved in the pathophysiology of malignancy, and several glycosylated compounds are currently used as biomarkers for cancer diagnosis and prognosis (33). In ovarian cancer, specific changes in glycosylation have been detected in tissue samples (34) and blood samples (35). In this study, we focused on a unique form of post-translational glycosylation, C-mannosylation.

The monomeric form of C-mannosylated tryptophan (CMW) is found in human blood and urine $(9,18)$. In contrast to the enzymatic pathway producing C-mannosylated $\operatorname{Trp}$ in proteins $(12,13,15)$, the metabolic pathway producing monomeric CMW in the cell has yet to be identified. Takahira et al (18) reported that CMW in blood could be a reliable biomarker for renal dysfunction, and several subsequent reports supported the utility of CMW as a diagnostic marker of renal damage (17-21). In addition, metabolomic profiling revealed that CMW in fasting blood is correlated with age and aging traits, such as lung function and bone mineral density (36). Lustgarten et al (37) also reported that CMW is a specific metabolite associated with elevated inflammation in older adults. In a serum metabolic profiling study of all-cause human mortality, CMW showed a statistically significant association with cardiovascular disease mortality (38). These findings demonstrate that blood CMW concentration could be a useful multi-functional biomarker for health and disease. Consistent with this notion, plasma CMW differentiated ovarian cancer from borderline or benign tumor with high sensitivity and specificity (Fig. 2A) and performed even better when combined with serum CA125 (Fig. 3). Furthermore, CA125 and CMW were uncorrelated (Fig. 2F), supporting the usefulness of combined evaluation for malignant ovarian cancer patients. High levels of CMW and CA125 in malignant ovarian cancer patients were also reduced following NAC and surgery (Fig. 4A, B) but further increased in a patient unresponsive to NAC (Fig. 4C). In this study, the tissue CMW status was not examined because the tissue samples were not available for the assays. Although no significant difference in tissue CMW status was observed between normal and cancerous ovarian tissues (Fig. 1B), further investigation for the tissue CMW status should be required to clarify the mechanism to affect the blood CMW status of cancer patients during NAC treatment. Furthermore, there have been no reports discussing on the half-life period of CMW in blood, and it was not clear whether the timing of the blood collection affects the tumor-related upregulation of blood CMW status in the patients. Thus, further investigation including basic researches is needed to clarify how the half-life period of $\mathrm{CMW}$ in blood is affected during the clinical treatment such as NAC. Together, these results suggest that measuring blood CMW could be a reliable approach to ovarian cancer diagnosis and prognosis. However, large-scale studies are required for confirmation and assessment of the utility of CMW measurement for distinguishing among malignant ovarian tumors of different histological subtypes and stages.

In our recent study, we examined the level of CMW in the blood and urine of C57BL/6 mice at 10 weeks $(n=5)$, and found that the concentration is apparently higher in urine $(30.7 \pm 10.2 \mu \mathrm{M})$ than in plasma $(0.099 \pm 0.017 \mu \mathrm{M})$, suggesting that CMW is highly excreted from the blood to urine in the kidney. We also found that CMW clearance was positively correlated with the creatinine clearance in normal and KK-Ay diabetic mice at 16 weeks (25). These results seem to be consistent with previous findings demonstrating that $\mathrm{CMW}$ is a possible marker to assess renal function in humans (17-21). As described above, urinary CMW is easy to measure because of its high excretion in urine, but the level may fluctuate in different urination conditions, such as polyuria and oliguria. By means of urinary CMW, a method to estimate the change of CMW in the whole body has yet to be established. Although 
patients with renal dysfunction were not included in this study, we actually observed high levels of plasma CMW in the patients with renal diseases (data not shown). Therefore, in this study, we focused on measuring CMW in blood rather than urine to assess the ovarian cancer patients. It is noteworthy that renal dysfunction can affect clinical assessment of CMW for ovarian cancer patients. Thus, further studies are also required for the assessment of the utility of CMW measurement to distinguish between ovarian tumors and other pathological conditions.

In ovarian cancer cells, upregulated indoleamine 2,3-dioxygenase, a critical enzyme of the kynurenine pathway, likely contributes to a decrease in Trp, resulting in immune tolerance by suppressing immune cell functions (39). Furthermore, serum Trp is significantly reduced in ovarian cancer patients compared to controls, suggesting that Trp metabolism is accelerated (40). Thus, it would be valuable to know whether these metabolic pathways are related to the metabolic regulation of CMW. However, no significant differences in CMW were detected between normal and cancerous ovarian tissues (Fig. 1B), indicating that increased blood CMW in ovarian tumor patients is not due simply to egress from cancer tissues and suggesting that other mechanisms related to cellular excretion or trafficking of CMW may be involved in the blood CMW elevation among ovarian cancer patients. We are currently conducting in vitro experiments using ovarian cancer cell lines to clarify the regulatory mechanisms responsible for the CMW increase in the blood of ovarian cancer patients. Moreover, it is of interest to know whether blood CMW has any specific biological function in the tumor cells or normal tissues of ovarian cancer patients.

Collectively, the present study demonstrates the feasibility of using blood CMW as a biomarker to detect ovarian malignant tumors. Further studies are warranted to confirm the utility of blood CMW for early diagnosis, staging, and treatment response.

\section{Acknowledgements}

Not applicable.

\section{Funding}

The present study was supported by Ministry of Education, Culture, Sports, Science and Technology of Japan Grants-inAid for Scientific Research Grant (grant no. JP16H06290).

\section{Availability of data and materials}

The datasets obtained and/or analyzed during the present study are available from the corresponding author on reasonable request.

\section{Authors' contributions}

NI and KI performed the experiments, and analyzed and interpreted the data. YIn, SS, SMi, SMa and YIt designed and performed the experiments, and analyzed the data. YIh conceived and designed the experiments, interpreted the data, and wrote the manuscript, and takes full responsibility for the manuscript. All authors read and reviewed the final manuscript.

\section{Ethics approval and consent to participate}

The present study was approved by The Ethics Committee of Wakayama Medical University Faculty of Medicine (authorization no. 1825). All of the patients in the present study provided written informed consent for the use of their plasma and tissue samples.

\section{Patient consent for publication}

Written informed consent for publication of the present report was obtained from the patient.

\section{Competing interests}

The authors declare that they have no competing interests.

\section{References}

1. Du Bois A and Pfisterer J: Future options for first-line therapy of advanced ovarian cancer. Int J Gynecol Cancer 15 (Suppl 1): S42-S50, 2005.

2. Siegel RL, Miller KD and Jemal A: Cancer statistics, 2015. CA Cancer J Clin 65: 5-29, 2015.

3. Heintz AP, Odicino F, Maisonneuve P, Quinn MA, Benedet JL, Creasman WT, Ngan HY, Pecorelli S and Beller U: Carcinoma of the ovary. FIGO 26th annual report on the results of treatment in gynecological cancer. Int J Gynaecol Obstet 95 (Suppl 1): S161-S192, 2006

4. Dodge JE, Covens AL, Lacchetti C, Elit LM, Le T, Devries-Aboud M and Fung-Kee-Fung M; Gynecology Cancer Disease Site Group: Preoperative identification of a suspicious adnexal mass: A systematic review and meta-analysis. Gynecol Oncol 126: 157-166, 2012.

5. Nam EJ, Yun MJ, Oh YT, Kim JW, Kim JH, Kim S, Jung YW, Kim SW and Kim YT: Diagnosis and staging of primary ovarian cancer: Correlation between PET/CT, Doppler US, and CT or MRI. Gynecol Oncol 116: 389-394, 2010.

6. Buamah P: Benign conditions associated with raised serum CA-125 concentration. J Surg Oncol 75: 264-265, 2000.

7. Buys SS, Partridge E, Black A, Johnson CC, Lamerato L, Isaacs C, Reding DJ, Greenlee RT, Yokochi LA, Kessel B, et al: Effect of screening on ovarian cancer mortality: The prostate, lung, colorectal and ovarian (PLCO) cancer screening randomized controlled trial. JAMA 305: 2295-2303, 2011.

8. Jacobs IJ, Menon U, Ryan A, Gentry-Maharaj A, Burnell M, Kalsi JK, Amso NN, Apostolidou S, Benjamin E, Cruickshank D, et al: Ovarian cancer screening and mortality in the UK collaborative trial of ovarian cancer screening (UKCTOCS): A randomized controlled trial. Lancet 387: 945-956, 2016.

9. Horiuchi K, Yonekawa O, Iwahara K, Kanno T, Kurihara T and Fujise Y: A hydrophilic tetrahydro-beta-carboline in human urine. J Biochem 115: 362-366, 1994.

10. Gutsche B, Grun C, Scheutzow D and Herderich M: Tryptophan glycoconjugates in food and human urine. Biochem J 343: 11-19, 1999.

11. Hofsteenge J, Müller DR, de Beer T, Löffler A, Richter WJ and Vliegenthart JF: New type of linkage between a carbohydrate and a protein: C-glycosylation of a specific tryptophan residue in human RNase Us. Biochemistry 33: 13524-13530, 1994.

12. Furmanek A and Hofsteenge J: Protein C-mannosylation: Facts and questions. Acta Biochim Pol 47: 781-789, 2000.

13. Shcherbakova A, Tiemann B, Buettner FF and Bakker H: Distinct C-mannosylation of netrin receptor thrombospondin type 1 repeats by mammalian DPY19L1 and DPY19L3. Proc Natl Acad Sci USA 114: 2574-2579, 2017.

14. Buettner FF, Ashikov A, Tiemann B, Lehle L and Bakker H: C. Elegans DPY-19 is a C-mannosyltransferase glycosylating thrombospondin repeats. Mol Cell 50: 295-302, 2013.

15. Niwa Y, Suzuki T, Dohmae N and Simizu S: Identification of DPY19L3 as the C-mannosyltransferase of R-spondin1 in human cells. Mol Biol Cell 27: 744-756, 2016. 
16. Ihara Y, Inai Y, Ikezaki M, et al: C-Mannosylation: Modification on tryptophan in celluar proteins. In: Taniguchi N, Endo T, Hart GW, et al, (eds). Glycoscience, biology and medicine Vol. 2, Springer Japan, 1091-1099, 2015

17. Sekula P, Dettmer K, Vogl FC, Gronwald W, Ellmann L, Mohney RP, Eckardt KU, Suhre K, Kastenmüller G, Oefner PJ and Köttgen A: From discovery to translation: Characterization of C-mannosyltryptophan and pseudouridine as markers of kidney function. Sci Rep 7: 17400, 2017.

18. Takahira R, Yonemura K, Yonekawa O, Iwahara K, Kanno T, Fujise Y and Hishida A: Tryptophan glycoconjugate as a novel marker of renal function. Am J Med 110: 192-197, 2001.

19. Yonemura K, Takahira R, Yonekawa O, Wada N and Hishida A The diagnostic value of serum concentrations of 2-(alphamannopyranosyl)-L-tryptophan for normal renal function. Kidney Int 65: 1395-1399, 2004.

20. Niewczas MA, Sirich TL, Mathew AV, Skupien J, Mohney RP, Warram JH, Smiles A, Huang X, Walker W, Byun J, et al: Uremic solutes and risk of end-stage renal disease in type 2 diabetes: Metabolomic study. Kidney Int 85: 1214-1224, 2014.

21. Solini A, Manca ML, Penno G, Pugliese G, Cobb JE and Ferrannini E: Prediction of declining renal function and albuminuria in patients with type 2 diabetes by metabolomics. J Clin Endocrinol Metab 101: 696-704, 2016.

22. Mizuta H, Kuga K, Suzuki T, Niwa Y, Dohmae N and Simizu S: C-mannosylation of R-spondin 2 activates Wnt/ $\beta$-catenin signaling and migration activity in human tumor cells. Int J Oncol 54: 2127-2138, 2019.

23. Li Y, Cao C, Jia W, Yu L, Mo M, Wang Q, Huang Y, Lim JM, Ishihara M, Wells L, et al: Structure of the F-spondin domain of mindin, an integrin ligand and pattern recognition molecule. EMBO J 28: 286-297, 2009.

24. Simon I, Liu Y, Krall KL, Urban N, Wolfert RL, Kim NW and McIntosh MW: Evaluation of the novel serum markers B7-H4 Spondin 2, and DcR3 for diagnosis and early detection of ovarian cancer. Gynecol Oncol 106: 112-118, 2007.

25. Sakurai S, Inai $Y$, Minakata S, Manabe S, Ito $Y$ and Ihara $Y$ : A novel assay for detection and quantification of C-mannosyl tryptophan in normal or diabetic mice. Sci Rep 9: 4675, 2019.

26. Manabe $S$ and Ito Y: Total synthesis of novel subclass of Glycoamino acid structure motif: C2- $\alpha$-D-C-Mannosyl-L-tryptophan. J Am Chem Soc 121: 9754-9755, 1999.

27. Burger RA, Brady MF, Bookman MA, Fleming GF, Monk BJ, Huang H, Mannel RS, Homesley HD, Fowler J, Greer BE, et al: Incorporation of bevacizumab in the primary treatment of ovarian cancer. N Engl J Med 365: 2473-2483, 2011.

28. Ledermann J, Harter P, Gourley C, Friedlander M, Vergote I, Rustin G, Scott CL, Meier W, Shapira-Frommer R, Safra T, et al: Olaparib maintenance therapy in patients with platinum-sensitive relapsed serous ovarian cancer: A preplanned retrospective analysis of outcomes by BRCA status in a randomized phase 2 trial. Lancet Oncol 15: 852-861, 2014.

29. Menon U, Talaat A, Rosenthal AN, Macdonald ND, Jeyerajah AR, Skates SJ, Sibley K, Oram DH and Jacobs IJ: Performance of ultrasound as a second line test to serum CA125 in ovarian cancer screening. BJOG 107: 165-169, 2000.
30. Rocconi RP, Matthews KS, Kemper MK, Hoskins KE, Huh WK and Straughn JM Jr: The timing of normalization of CA-125 levels during primary chemotherapy is predictive of survival in patients with epithelial ovarian cancer. Gynecol Oncol 114: 242-245, 2009.

31. Drescher CW, Shah C, Thorpe J, O'Briant K, Anderson GL, Berg CD, Urban N and McIntosh MW: Longitudinal screening algorithm that incorporates change over time in CA125 levels identifies ovarian cancer earlier than a single-threshold rule. J Clin Oncol 31: 387-392, 2013

32. Menon U, Gentry-Maharaj A, Hallett R, Ryan A, Burnell M, Sharma A, Lewis S, Davies S, Philpott S, Lopes A, et al: Sensitivity and specificity of multimodal and ultrasound screening for ovarian cancer, and stage distribution of detected cancers: Results of the prevalence screen of the UK Collaborative trial of ovarian cancer screening (UKCTOCS). Lancet Oncol 10: 327-340, 2009.

33. Adamczyk B, Tharmalingam T and Rudd PM: Glycans as cancer biomarkers. Biochim Biophys Acta 1820: 1347-1353, 2012.

34. Anugraham M, Jacob F, Everest-Dass AV, Schoetzau A, Nixdorf S, Hacker NF, Fink D, Heinzelmann-Schwarz V and Packer NH: Tissue glycomics distinguish tumour sites in women with advanced serous adenocarcinoma. Mol Oncol 11: 1595-1615, 2017.

35. Biskup K, Braicu EI, Sehouli J, Fotopoulou C, Tauber R, Berger $M$ and Blanchard V: Serum glycome profiling: A biomarker for diagnosis of ovarian cancer. J Proteome Res 12: 4056-4063, 2013.

36. Menni C, Kastenmüller G, Petersen AK, Bell JT, Psatha M, Tsai PC, Gieger C, Schulz H, Erte I, John S, et al: Metabolomic markers reveal novel pathways of ageing and early development in human populations. Int J Epidemiol 42: 1111-1119, 2013.

37. Lustgarten MS and Fielding RA: Metabolites related to renal function, immune activation, and carbamylation are associated with muscle composition in older adults. Exp Gerontol 100: 1-10, 2017.

38. Huang J, Weinstein SJ, Moore SC, Derkach A, Hua X, Liao LM, Gu F, Mondul AM, Sampson JN and Albanes D: Serum metabolomic profiling of all-cause mortality: A prospective analysis in the alpha-tocopherol, beta-carotene cancer prevention (ATBC) study cohort. Am J Epidemiol 187: 1721-1732, 2018

39. Tanizaki Y, Kobayashi A, Toujima S, Shiro M, Mizoguchi M, Mabuchi Y, Yagi S, Minami S, Takikawa $O$ and Ino $K$ : Indoleamine 2,3-dioxygenase promotes peritoneal metastasis of ovarian cancer by inducing an immunosuppressive environment. Cancer Sci 105: 966-973, 2014

40. Schroecksnadel K, Winkler C, Fuith LC and Fuchs D: Tryptophan degradation in patients with gynecological cancer correlates with immune activation. Cancer Lett 223: 323-329, 2005.

This work is licensed under a Creative Commons

Attribution-NonCommercial-NoDerivatives 4.0 International (CC BY-NC-ND 4.0) License. 